\section{EACS}

How to cite this article: Mello et al. Impact of equine assisted therapy on the cardiovascular parameters of the elderly. ABCS Health Sci. 2021;46:e021216. https://doi.org/10.7322/abcshs.2019161.1430

Received: Dec 20, 2019

Revised: Aug 14, 2020

Approved: Nov 10, 2020

Corresponding author: Ana Paula Espindula - Universidade Federal do Triângulo Mineiro - Rua Frei Paulino, 30 Nossa Senhora da Abadia - CEP: 38025-180 - Uberaba (MG), Brazil -

Email: anapaulaespindula@yahoo.com.br

Declaration of interests: nothing to declare Funding: CNPq, FAPEMIG, FUNEPU, APAE

This is an open access article distributed under the terms of the Creative Commons Attribution License

(C) 2021 The authors

\title{
Impact of equine assisted therapy on the cardiovascular parameters of the elderly
}

\author{
Edneia Correa Mello', Lunna Honorato Diniz', Janaine Brandão Lage1, \\ Valéria Silva Monteiro², Mariane Fernandes Ribeiro ${ }^{1}$, Rodrigo César Rosa ${ }^{3}$, \\ Domingos Emanuel Bevilacqua Junior ${ }^{1}$, Fabrizio Antônio Gomide Cardoso ${ }^{3}$, \\ Vicente de Paula Antunes Teixeira ${ }^{1}$, Ana Paula Espindula ${ }^{1,3}$ \\ ${ }^{1}$ Programa de Pós-Graduação em Ciências da Saúde em Patologia Humana, Universidade \\ Federal do Triângulo Mineiro (UFTM) - Uberaba (MG), Brazil \\ ²Educação Física, Associação de Pais e Amigos dos Excepcionais (APAE) - Uberaba (MG), Brazil \\ ${ }^{3}$ Disciplina de Anatomia Humana, UFTM - Uberaba (MG), Brazil
}

\begin{abstract}
Introduction: Physical activity programs in the elderly aim to prevent and/or reduce the functional decline resulting from the senescence process. Several studies provide evidence that Equine Assisted Therapy (EAT) is an effective means of improving health. However, studies addressing the effects of EATon the cardiovascular system are scarce. Objective: To evaluate the effect of Equine Assisted Therapyon the cardiovascular responses of participants divided into two groups: normotensive and hypertensive. Methods: Twenty individuals participated, aged between 60 and 79 years, divided into three groups: Normotensive Group $(n=14)$, Hypertensive Group $(n=6)$ and all participants, Total Group $(n=20)$. The Omron ${ }^{\circledR}$ HEM 742 blood pressure monitor was used to measure blood pressure and the Gerathem ${ }^{\circledR}$ portable finger oximeter to measure heart rate and blood oxygen saturation. Ten visits were made once a week, lasting 30 minutes. Results: Between the first and tenth interventions, there was a reduction in blood pressure and heart rate, with statistical significance of systolic blood pressure for the Hypertensive Group ( $p=0.0478$ ), the Total Group ( $p=0.0201$ ) and diastolic pressure for the Total Group $(\mathrm{p}=0.0421)$. There was also a statistically significant difference in systolic blood pressure and heart rate during some visits. Blood oxygen saturation increased after the intervention, but without statistical significance. Conclusion: Equine Assisted Therapy can promote a reduction in blood pressure in the elderly, especially hypertensive individuals.
\end{abstract}

Keywords: equine-assisted therapy; aged; blood pressure; heart rate.

\section{INTRODUCTION}

The aging process is progressive and dynamic, providing innumerable morphological, functional, biochemical, and psychological changes, resulting in the loss of individual capacity, which can lead to vulnerability and incidence of diseases. Population aging is a universal and irreversible phenomenon ${ }^{1,2}$.

Cardiovascular disease is the leading cause of adult death in the world, greatly increasing the incidence with the aging process. In the elderly with more than 75 years, it can reach $50 \%$, being considered the main cause of morbimortality in the geriatric population ${ }^{3,4}$. Systemic Arterial Hypertension is considered the main modifiable 
risk factor for cardiovascular diseases. Blood pressure is directly related to age, with prevalence of $60 \%$ for the age group over 65 years. In fact, Systemic Arterial Hypertension is a chronic non-transmissible disease, more prevalent among the elder$1 y^{5,6}$. It is a significant and often asymptomatic chronic disease, which requires optimal control and persistent adherence to prescribed medication to reduce the risks of cardiovascular, cerebrovascular, and renal disease. Multiple health organizations, including the American College of Preventive Medicine, emphasize physical activity, such as non-drug strategies, for blood pressure management ${ }^{7}$.

The American Heart Association issued scientific statement reporting that contact with animals can reduce the risk of cardiovascular disease, dyslipidemia, obesity and improve the functionality, autonomy, and survival of people with cardiovascular disease $^{8}$. Animal-Assisted Therapy is not a practice for replacing conventional therapies and treatments, but a complement, a new line of therapy and research, recognized in several countries, which takes into account the influence of emotions on human health. Animals of different species can be used in this therapy, but the most commonly used are dogs and horses, because they are generally docile $\mathrm{e}^{9-11}$.

Equine-Assisted Therapy (EAT) is an effective way of improving many measures of physical health ${ }^{12,13}$. However, studies approaching the effects of EAT on the cardiovascular system are scarce. A review of the literature concluded that cardiovascular responses to EAT remain poorly defined. At present, there is insufficient evidence to suggest that EAT has a residual or training effect similar to the generally expected from training to improve cardiovascular fitness ${ }^{14}$. The cardiovascular fitness is a central measure of physical fitness and an independent predictor of chronic health conditions. So, it is a tremendous value for clinicians to understand cardiovascular responses promoted by EAT. This may lead to a greater demand for EAT to be recognized as beneficial by health insurance providers and ultimately result in it becoming more affordable and accessible ${ }^{15}$.

In this context, the primary objective of this study was to evaluate the effect of EAT on cardiovascular responses in older adults. The secondary objective was to evaluate the effect of EAT on the cardiovascular responses of participants divided into two groups: normotensive and hypertensive. For this, data were collected on systolic and diastolic blood pressure, heart rate and partial oxygen saturation. The data were used to evaluate the cardiovascular response in two situations: between the first and the tenth intervention and during the EAT session. It is known that uncontrolled hypertension is a contraindication for EAT and that older adults usually have lower levels of oxygen saturation than younger adults. When the hypoxia process starts, it accelerates and the condition quickly becomes more serious ${ }^{16,17}$. Therefore, the constant assessment of cardiovascular parameters during sessions keeps EAT interventions safer, especially for the geriatric population. We hypothesized that EAT provides positive cardiovascular responses for older adults, whether they are normotensive or hypertensive.

\section{METHODS}

This is a longitudinal, quantitative and descriptive study. The project was evaluated, and approved by the Research Ethics Committee (CEP) of the Universidade Federal do Triângulo Mineiro (UFTM) - under the protocol number 690.039 on $13 / 02 / 2014$, by the UFTM Ethical Committee on the Use of Animals under protocol no. 266/2017on 21/07/2017 and by the Brazilian Registry of Clinical Trials (ReBEC) under protocol RBR$2 \mathrm{kw} 6 \mathrm{p} 9$. The evaluation methods and intervention protocols used in this study adhered to the norms of Resolution 466/12 of the Brazilian National Health Council on Research Involving Human Beings and the Law 11.794/08 Decree 6.899/09 by the National Council for Animal Control and Experimentation (CONCEA). Participants in this study provided written informed consent by signing a Free and Informed Consent Form.

\section{Participants}

In a sample of 90 individuals evaluated and following the inclusion and exclusion criteria, 20 individuals were selected. Inclusion and exclusion criteria were determined through clinical examination and anamnesis, in which information was obtained regarding personal data, lifestyle, clinical history, diseases and use of medications. Individuals with unmatched age (60 to 80 years), gait impairment, severe spinal disorders, hip dislocation, uncontrollable fear of horses, and/or physical training practitioners were excluded. Hypertension is a relative contraindication for EAT. However, frequent episodes of high blood pressure make it a contraindication to therapy. Therefore, individuals who had uncontrolled hypertension were not included in the study, which justifies the lower number of HG.As a discontinuity criterion, individuals who had more consecutive absences and/or did not complete the ten sessions were excluded. All participants started the practice of EAT with the Project. Participants were allocated into three groups: the normotensive group (NG) formed by 14 individuals, the hypertensive group (HG) by six individuals and the total group (TG) by 20 individuals, all participants. Normal blood pressure was defined as $<120 / 80 \mathrm{mmHg}$ and hypertension as $\geq 130 / 80 \mathrm{~mm} \mathrm{Hg}^{7}$. Hypertensive individuals, who had previously been diagnosed, used their usual blood pressure medications. One individual used angiotensin-converting enzyme (Captopril), two individuals used angiotensin II receptor blockers (Losartan, Valsartan), one individual used beta-blocker (Atenolol), one individual used diuretic (Hydrochlorothiazide) and one individual used 
angiotensin II receptor blockers with diuretic (Benicar Olmesartan Medoxomil with Hydrochlorothiazide)

The anthropometric variables were evaluated by means of a standardization of the International Society for the Advancement of Cineanthropometry (ISAK). Total body mass (TBM) and height were measured using a Filizola ${ }^{\circledR}$ balance with a precision of 100 grams with stadiometer coupled with a precision of $0.5 \mathrm{~cm}$. The body mass index (BMI) was calculated using the previously obtained anthropometric measures, according to a predetermined equation ${ }^{18}$. The characterization of each group is in Table 1.

\section{Interventions}

The sessions were held at the Centro de Equoterapia Dr. Guerra of the Associação de Pais e Amigos dos Excepcionais (APAE) in the city of Uberaba, Minas Gerais, Brazil. The center of EAT contains an appropriate covered area, with rings, bays, saddlery, and platform of access. The proposed intervention protocol was in accordance with the experience of the hippotherapy center ${ }^{19,20}$. The ten sessions were performed with the horse at a pace, for 30 minutes, once a week. Three horses were used randomly, aged between 20, 18 and 9 years and with height of 1.56, 1.60 and $1.62 \mathrm{~m}$, respectively. The riding equipment used was saddle, with the feet in the stirrups in the first 15 minutes, and with the feet out of the stirrups in the remaining 15 minutes. No type of activity or exercise was performed by the participant during the session, because the objective was to evaluate the effect of the three-dimensional movement provided by the equine. The sessions were conducted only by examiners previously qualified by the National Equine Therapy Association ${ }^{21}$.

\section{Evaluation procedures}

The validated and properly calibrated Omron ${ }^{\circledR}$ HEM 742 pressure monitor was used to measure blood pressure. The arm was supported at the heart level, with the palm facing upwards and the cuff placed two to three $\mathrm{cm}$ above the cubital fossa and centered on the brachial artery. The Gerathem ${ }^{\circledast}$ portable fingertip pulse oximeter, positioned on the left index finger, was used to measure heart rate and partial oxygen saturation. The data collection was performed in five moments: at initial rest (Pre-EAT), in the first

Table 1: Characterization of the normotensive, hypertensive and total group, age (years), height $(\mathrm{m})$, weight $(\mathrm{Kg})$ and Body Mass Index (BMI - Kg/m²), (Mean \pm SDM).

\begin{tabular}{|l|c|c|c|c|}
\hline Group & $\begin{array}{c}\text { Age } \\
(\text { years })\end{array}$ & $\begin{array}{c}\text { Height } \\
(\mathrm{m})\end{array}$ & $\begin{array}{c}\text { Weight } \\
(\mathrm{Kg})\end{array}$ & $\begin{array}{c}\mathrm{BMI} \\
\left(\mathrm{Kg} / \mathrm{m}^{2}\right)\end{array}$ \\
\hline $\begin{array}{l}\text { Normotensive } \\
(n=14)\end{array}$ & $65.14 \pm 6.84$ & $1.58 \pm 0.08$ & $62.35 \pm 10.07$ & $25.06 \pm 3.87$ \\
\hline $\begin{array}{l}\text { Hypertensive } \\
(n=6)\end{array}$ & $67.33 \pm 5.07$ & $1.52 \pm 0.06$ & $61.73 \pm 15.75$ & $26.35 \pm 4.74$ \\
\hline Total $(\mathrm{n}=20)$ & $65.80 \pm 6.30$ & $1.56 \pm 0.08$ & $62.17 \pm 11.61$ & $25.45 \pm 4.07$
\end{tabular}

minute of the session (1st minute), fifteenth minute (15th minute), in the thirty minute (30th minute) and ten minutes after the session (Post-EAT).

\section{Data Analysis}

The results were obtained through the descriptive analysis for each variable. Fordata with two variables, the $t$ test for paired samples was used. For tests with more than two variables, Repeated Measures ANOVA, post-hoc Bonferroni were used. The analyses were conducted using the GraphPad Prism ${ }^{\circledR}$ software (version 5.0, San Diego, USA), considering a significance level of $5 \%$ and a confidence interval of $95 \%$.

\section{RESULTS}

There were reductions in blood pressure and heart rate between the first and last intervention, with statistical significance of systolic blood pressure for HG ( $\mathrm{p}=0.0478)$, TG $(\mathrm{p}=0.0201)$ and diastolic blood pressure for TG $(\mathrm{p}=0.0421)$. The three groups had the same ideal systolic, $120 \mathrm{mmHg}$, and diastolic blood pressures, less than $80 \mathrm{mmHg}$. Partial oxygen saturation increased, however without statistical difference (Table 2).

There were statistical differences in systolic blood pressure on day 2 and diastolic blood pressure on days 1, 5 and 6 for NG (Table 3).

There were significant reductions in systolic blood pressure on day 4 and diastolic blood pressure on day 9 for HG (Table 4).

There were statistical differences in systolic blood pressure on day 1 and diastolic blood pressure on days 1 and 5 for TG (Table 5).

There were significant reductions in heart rate on days 2,6 , 7 and 9 for NG, on days 3 and 4 for HG, on days 2, 4 and 6 for TG, Table 6.

Table 2: Systolic and diastolic blood pressure, heart rate and partial oxygen saturation of Normotensive, Hypertensive and Total Group Pre-EAT of the first and last intervention (Mean \pm SEM and statistical significance ${ }^{*} p<0.05$ ).

\begin{tabular}{|l|c|c|c}
\multirow{2}{*}{ Systolic Blood Pressure } & Session 1 & Session 10 & \multirow{2}{*}{$p$} \\
\cline { 2 - 3 } Normotensive & Pre-EAT & Pre-EAT & \\
\hline Hypertensive & $124.3 \pm 3.43$ & $120.0 \pm 2.77$ & 0.2536 \\
\hline Total & $138.3 \pm 6.54$ & $120.0 \pm 3.65$ & $0.0478^{*}$ \\
\hline Diastolic Blood Pressure & $129.0 \pm 3.31$ & $120.0 \pm 2.17$ & $0.0201^{*}$ \\
\hline Normotensive & $77.14 \pm 2.85$ & $70.71 \pm 2.86$ & 0.0951 \\
\hline Hypertensive & $83.33 \pm 3.33$ & $73.33 \pm 5.57$ & 0.1438 \\
\hline Total & $78.00 \pm 2.24$ & $72.00 \pm 2.47$ & $0.0421^{*}$ \\
\hline Heart Rate & Pre-EAT & Post-EAT & $p$ \\
\hline Normotensive & $78.57 \pm 2.64$ & $77.64 \pm 2.97$ & 0.7904 \\
\hline Hypertensive & $78.17 \pm 4.55$ & $73.33 \pm 6.84$ & 0.3401 \\
\hline Total & $78.35 \pm 2.22$ & $78.50 \pm 2.57$ & 0.9572 \\
\hline Oxygen Saturation & Pre-EAT & Post-EAT & $p$ \\
\hline & $96.45 \pm 0.49$ & $96.80 \pm 0.22$ & 0.4392
\end{tabular}


Table 3: Systolic and diastolic blood pressure of Normotensive Group Pre-, during [first ( $\left.1^{\text {st }}\right)$, fifteenth $\left(15^{\text {th }}\right)$, thirtieth $\left(30^{\text {th }}\right)$ minute] and PostEAT (Mean \pm SEM and same letter is statistical significance ${ }^{*} p<0.05,{ }^{* *} p<0.01$ ).

\begin{tabular}{|c|c|c|c|c|c|}
\hline \multicolumn{6}{|c|}{ Systolic } \\
\hline Day & Pre-EAT & $1^{\text {st }}$ minute & $15^{\text {th }}$ minute & $30^{\text {th }}$ minute & Post-EAT \\
\hline 1 & $124.3 \pm 3.43$ & $132.1 \pm 3.65$ & $124.3 \pm 4.28$ & $120.7 \pm 4.25$ & $120.7 \pm 3.70$ \\
\hline 2 & $121.4 \pm 4.55$ & $127.9 \pm 4.34$ & $122.9 \pm 4.37$ & $120.7 \pm 4.12^{\mathrm{a} *}$ & $132.1 \pm 3.94^{\mathrm{a} *}$ \\
\hline 3 & $122.9 \pm 3.54$ & $122.9 \pm 4.11$ & $122.9 \pm 1.63$ & $125.0 \pm 3.10$ & $119.3 \pm 3.22$ \\
\hline 4 & $120.0 \pm 3.31$ & $123.6 \pm 3.86$ & $123.6 \pm 4.00$ & $122.9 \pm 5.18$ & $117.1 \pm 4.73$ \\
\hline 5 & $122.9 \pm 3.21$ & $122.9 \pm 4.50$ & $118.6 \pm 3.29$ & $122.1 \pm 2.80$ & $116.4 \pm 2.69$ \\
\hline 6 & $123.6 \pm 4.00$ & $120.7 \pm 3.70$ & $119.3 \pm 3.22$ & $120.0 \pm 2.96$ & $116.4 \pm 3.86$ \\
\hline 7 & $120.0 \pm 2.77$ & $118.6 \pm 2.05$ & $117.9 \pm 2.38$ & $117.1 \pm 3.84$ & $118.6 \pm 2.53$ \\
\hline 8 & $116.4 \pm 2.25$ & $114.3 \pm 2.50$ & $117.1 \pm 2.85$ & $117.1 \pm 2.20$ & $117.1 \pm 2.44$ \\
\hline 9 & $120.7 \pm 2.86$ & $117.1 \pm 2.65$ & $115.7 \pm 4.53$ & $115.7 \pm 3.58$ & $121.4 \pm 2.93$ \\
\hline 10 & $120.0 \pm 2.77$ & $124.3 \pm 2.27$ & $119.3 \pm 3.54$ & $120.0 \pm 2.77$ & $119.3 \pm 3.54$ \\
\hline \multicolumn{6}{|c|}{ Diastolic } \\
\hline Day & Pre-EAT & $1^{\text {st }}$ minute & $15^{\text {th }}$ minute & $30^{\text {th }}$ minute & Post-EAT \\
\hline 1 & $77.14 \pm 2.85^{\mathrm{a} \star \star}$ & $80.71 \pm 2.45$ & $76.43 \pm 2.69$ & $75.71 \pm 3.09$ & $69.79 \pm 2.10^{\mathrm{a} * \star}$ \\
\hline 2 & $80.71 \pm 4.74$ & $80.00 \pm 2.77$ & $78.57 \pm 2.74$ & $77.14 \pm 3.04$ & $78.57 \pm 2.74$ \\
\hline 3 & $77.86 \pm 2.99$ & $78.57 \pm 2.74$ & $79.29 \pm 3.22$ & $77.86 \pm 2.80$ & $73.57 \pm 2.69$ \\
\hline 4 & $75.00 \pm 2.51$ & $75.00 \pm 2.51$ & $79.29 \pm 3.05^{a \star}$ & $76.43 \pm 4.14$ & $70.71 \pm 2.45^{\mathrm{a} *}$ \\
\hline 5 & $69.29 \pm 1.95^{a, b * *}$ & $75.71 \pm 2.27$ & $79.29 \pm 2.86^{\mathrm{a} * *}$ & $77.86 \pm 1.8^{\mathrm{b} * \star}$ & $72.86 \pm 2.65$ \\
\hline 6 & $74.29 \pm 2.71$ & $75.00 \pm 3.27$ & $77.86 \pm 2.60^{\mathrm{a} \star}$ & $77.86 \pm 1.86^{b *}$ & $69.29 \pm 1.95^{\mathrm{a}, \mathrm{b} *}$ \\
\hline 7 & $72.14 \pm 2.99$ & $74.29 \pm 3.88$ & $75.00 \pm 2.72$ & $76.43 \pm 3.24$ & $72.14 \pm 2.14$ \\
\hline 8 & $77.86 \pm 3.50$ & $74.29 \pm 2.71$ & $74.29 \pm 2.27$ & $75.71 \pm 2.27$ & $70.71 \pm 2.86$ \\
\hline 9 & $70.71 \pm 2.21$ & $76.43 \pm 3.07$ & $75.71 \pm 2.91$ & $77.86 \pm 2.99$ & $77.14 \pm 2.85$ \\
\hline 10 & $70.71 \pm 2.86$ & $76.43 \pm 3.24$ & $72.86 \pm 3.69$ & $77.86 \pm 3.17$ & $73.57 \pm 3.57$ \\
\hline
\end{tabular}

Table 4: Systolic and diastolic blood pressure of Hypertensive Group Pre-, during [first $\left(1^{\text {st }}\right)$, fifteenth $\left(15^{\text {th }}\right)$, thirtieth $\left(30^{\text {th }}\right)$ minute] and PostEAT (Mean \pm SEM and same letter is statistical significance ${ }^{*} p<0.05,{ }^{\star *} p<0.01$ ).

\begin{tabular}{|c|c|c|c|c|c|}
\hline \multicolumn{6}{|c|}{ Systolic } \\
\hline Day & Pre-EAT & $1^{\text {st }}$ minute & $15^{\text {th }}$ minute & $30^{\text {th }}$ minute & Post-EAT \\
\hline 1 & $138.3 \pm 6.54$ & $138.3 \pm 11.08$ & $136.7 \pm 11.74$ & $136.7 \pm 9.18$ & $130.0 \pm 7.30$ \\
\hline 2 & $133.3 \pm 6.14$ & $141.7 \pm 10.46$ & $138.3 \pm 11.95$ & $138.3 \pm 9.09$ & $126.7 \pm 4.94$ \\
\hline 3 & $130.0 \pm 10.33$ & $131.7 \pm 8.72$ & $133.3 \pm 10.85$ & $121.7 \pm 7.49$ & $126.7 \pm 10.85$ \\
\hline 4 & $151.7 \pm 10.78$ & $155.0 \pm 8.06^{\mathrm{a} \star}$ & $138.3 \pm 7.49$ & $135.0 \pm 8.06$ & $133.3 \pm 6.14^{\mathrm{a} *}$ \\
\hline 5 & $125.0 \pm 8.06$ & $131.7 \pm 7.92$ & $125.0 \pm 6.70$ & $130.0 \pm 8.16$ & $128.3 \pm 6.00$ \\
\hline 6 & $113.3 \pm 6.66$ & $125.0 \pm 5.62$ & $118.3 \pm 6.54$ & $123.3 \pm 7.60$ & $118.3 \pm 9.45$ \\
\hline 7 & $116.7 \pm 11.45$ & $121.7 \pm 7.03$ & $128.3 \pm 4.01$ & $118.3 \pm 9.09$ & $130.0 \pm 3.65$ \\
\hline 8 & $120.0 \pm 11.25$ & $131.7 \pm 10.46$ & $126.7 \pm 8.81$ & $125.0 \pm 7.63$ & $125.0 \pm 4.28$ \\
\hline 9 & $123.3 \pm 11.74$ & $126.7 \pm 9.54$ & $125.0 \pm 8.06$ & $130.0 \pm 7.30$ & $125.0 \pm 8.46$ \\
\hline 10 & $120.0 \pm 3.65$ & $126.7 \pm 4.21$ & $121.7 \pm 7.03$ & $116.7 \pm 4.94$ & $115.0 \pm 8.46$ \\
\hline \multicolumn{6}{|c|}{ Diastolic } \\
\hline Day & Pre-EAT & $1^{\text {st }}$ minute & $15^{\text {th }}$ minute & $30^{\text {th }}$ minute & Post-EAT \\
\hline 1 & $83.33 \pm 3.33$ & $81.67 \pm 4.77$ & $81.67 \pm 6.00$ & $81.67 \pm 7.49$ & $81.67 \pm 4.77$ \\
\hline 2 & $73.33 \pm 4.21$ & $80.00 \pm 6.32$ & $81.67 \pm 6.00$ & $78.33 \pm 6.54$ & $75.00 \pm 5.00$ \\
\hline 3 & $73.33 \pm 4.21$ & $75.00 \pm 3.41$ & $73.33 \pm 4.94$ & $66.67 \pm 4.21$ & $71.67 \pm 6.00$ \\
\hline 4 & $80.00 \pm 6.32$ & $85.00 \pm 8.46$ & $75.00 \pm 5.00$ & $80.00 \pm 5.16$ & $77.33 \pm 4.05$ \\
\hline 5 & $66.67 \pm 6.14$ & $73.33 \pm 4.94$ & $76.67 \pm 7.14$ & $75.00 \pm 5.62$ & $68.33 \pm 4.01$ \\
\hline 6 & $66.67 \pm 4.94$ & $65.00 \pm 5.00$ & $65.00 \pm 5.00$ & $71.67 \pm 4.01$ & $70.00 \pm 5.77$ \\
\hline 7 & $58.33 \pm 4.77$ & $68.33 \pm 7.03$ & $75.00 \pm 5.62$ & $71.67 \pm 6.00$ & $76.67 \pm 5.57$ \\
\hline 8 & $73.33 \pm 9.18$ & $83.33 \pm 6.66$ & $71.67 \pm 4.77$ & $75.00 \pm 6.70$ & $70.00 \pm 8.56$ \\
\hline 9 & $78.33 \pm 7.03$ & $86.67 \pm 4.94$ & $81.67 \pm 6.00$ & $88.33 \pm 6.00^{\mathrm{a}^{*}}$ & $73.33 \pm 7.14^{\mathrm{a}^{*}}$ \\
\hline 10 & $73.33 \pm 5.57$ & $80.00 \pm 6.32$ & $70.00 \pm 3.65$ & $70.00 \pm 5.16$ & $73.33 \pm 6.66$ \\
\hline
\end{tabular}


Table 5: Systolic and diastolic blood pressure of Total Group Pre-. during [first $\left(1^{\text {st }}\right)$. fifteenth $\left(15^{\text {th }}\right)$. thirtieth $\left(30^{\text {th }}\right)$ minute] and Post- EAT (Mean \pm SEM and same letter is statistical significance ${ }^{*} p<0.05 .{ }^{* *} p<0.01$ ).

\begin{tabular}{|c|c|c|c|c|c|}
\hline Day & Pre-EAT & $1^{\text {st }}$ minute & $15^{\text {th }}$ minute & $30^{\text {th }}$ minute & Post-EAT \\
\hline 1 & $129.0 \pm 3.31^{\mathrm{a}^{*} \mathrm{~b}^{* *}}$ & $135.5 \pm 3.87$ & $129.0 \pm 4.52$ & $126.0 \pm 4.25^{\mathrm{a}^{*}}$ & $123.5 \pm 3.42^{\mathrm{b}^{* *}}$ \\
\hline 2 & $125.0 \pm 3.80$ & $133.0 \pm 4.41$ & $128.5 \pm 4.71$ & $126.5 \pm 4.24$ & $130.5 \pm 3.11$ \\
\hline 3 & $125.0 \pm 3.87$ & $127.5 \pm 4.46$ & $128.0 \pm 3.81$ & $124.5 \pm 3.03$ & $122.0 \pm 3.88$ \\
\hline 4 & $129.0 \pm 4.52$ & $132.5 \pm 4.34$ & $128.5 \pm 4.05$ & $129.0 \pm 5.32$ & $122.5 \pm 4.03$ \\
\hline 5 & $123.2 \pm 3.50$ & $126.3 \pm 4.84$ & $122.1 \pm 4.43$ & $126.3 \pm 5.14$ & $121.1 \pm 3.14$ \\
\hline 6 & $120.5 \pm 3.803$ & $123.0 \pm 3.25$ & $121.5 \pm 2.32$ & $120.0 \pm 3.16$ & $120.5 \pm 2.34$ \\
\hline 7 & $119.5 \pm 3.66$ & $122.0 \pm 3.12$ & $121.5 \pm 2.32$ & $120.0 \pm 3.16$ & $121.0 \pm 2.39$ \\
\hline 8 & $118.0 \pm 3.44$ & $120.5 \pm 3.87$ & $120.5 \pm 3.36$ & $120.0 \pm 2.81$ & $120.0 \pm 2.17$ \\
\hline 9 & $121.5 \pm 3.85$ & $119.5 \pm 3.43$ & $118.0 \pm 3.94$ & $119.5 \pm 3.58$ & $121.5 \pm 3.18$ \\
\hline 10 & $121.0 \pm 2.16$ & $126.5 \pm 1.95$ & $122.0 \pm 3.12$ & $120.5 \pm 2.56$ & $121.0 \pm 3.47$ \\
\hline Day & Pre-EAT & $1^{\text {st }}$ minute & $15^{\text {th }}$ minute & $30^{\text {th }}$ minute & Post-EAT \\
\hline 1 & $78.00 \pm 2.24^{a^{*}}$ & $81.00 \pm 2.16$ & $79.00 \pm 2.39$ & $78.00 \pm 2.95$ & $73.35 \pm 2.34^{a^{*}}$ \\
\hline 2 & $79.00 \pm 3.47$ & $81.00 \pm 2.39$ & $80.50 \pm 2.34$ & $78.00 \pm 2.67$ & $78.00 \pm 2.24$ \\
\hline 3 & $76.50 \pm 2.43$ & $77.50 \pm 2.16$ & $79.00 \pm 2.60$ & $76.00 \pm 2.55$ & $74.00 \pm 2.44$ \\
\hline 4 & $77.00 \pm 2.52$ & $78.00 \pm 2.95$ & $80.00 \pm 3.07$ & $79.00 \pm 3.62$ & $73.20 \pm 2.30$ \\
\hline 5 & $70.00 \pm 2.29^{a^{a} b, c^{* * * *}}$ & $76.50 \pm 2.43^{a^{*}}$ & $80.00 \pm 3.24^{b^{* *}, d^{* *}}$ & $79.00 \pm 2.60^{c^{* * *}, e^{*}}$ & $72.50 \pm 2.16^{d^{* *} \cdot e^{*}}$ \\
\hline 6 & $69.50 \pm 2.76$ & $75.00 \pm 3.44$ & $75.50 \pm 2.23$ & $77.00 \pm 2.62$ & $73.50 \pm 2.20$ \\
\hline 7 & $119.5 \pm 3.66$ & $122.0 \pm 3.12$ & $121.5 \pm 2.32$ & $120.0 \pm 3.16$ & $121.0 \pm 2.39$ \\
\hline 8 & $77.00 \pm 3.41$ & $78.50 \pm 2.74$ & $75.00 \pm 2.46$ & $77.00 \pm 2.72$ & $71.50 \pm 2.92$ \\
\hline 9 & $73.00 \pm 2.62$ & $78.50 \pm 2.74$ & $77.00 \pm 2.72$ & $80.50 \pm 2.85$ & $76.00 \pm 2.84$ \\
\hline 10 & $72.50 \pm 2.50$ & $77.50 \pm 2.89$ & $73.50 \pm 3.10$ & $76.50 \pm 2.74$ & $74.50 \pm 2.854$ \\
\hline
\end{tabular}

\section{DISCUSSION}

The results of the present study show that the EAT promoted a reduction in blood pressure and a low oscillation of the heart rate, which characterizes the intervention as a light physical activity and without risk for hypertensive patients, and maintains the oxygen saturation values within normal limits. These findings validate our hypothesis that EAT provides positive cardiovascular responses for older adults, whether they are normotensive or hypertensive. To our knowledge, this is the first study to compare blood pressure measurements in normotensive and hypertensive participants.

Over the life course, the heart progressively remodels. Typical age-related cardiac remodeling involves increasing left ventricular wall thickness, decreasing left ventricular dimensions, and increasing concentricity, all of which are associated with risk factors and incidence of adverse cardiovascular events, such as coronary artery disease, heart attack, and stroke. Age-related changes in vascular function generally include increasing endothelial dysfunction and arterial stiffness, accompanied by increasing systolic blood pressure and pulse pressure ${ }^{22,23}$. In elderly individuals, it is common for there to be abnormally high blood pressure during systole and normal blood pressure during diastole, isolated systolic hypertension ${ }^{24}$. At rest, the older heart works the same way as a younger heart, but the heart rate is slightly lower. In addition, during exercise, the heart rate of the elderly does not increase as much as in younger people ${ }^{25}$. Many of the effects of aging on the heart and blood vessels can be reduced by regular exercise. Exercise helps maintain cardiovascular capacity and fitness as people get older ${ }^{26,27}$.

The results of the current study show a significant reduction in blood pressure and heart rate after older adults participated in EAT sessions for 10 consecutive weeks. These results are in accordance with the guidelines that emphasize several forms of physical activity such as non-drug strategies for blood pressure management ${ }^{7}$. Moreover, these results corroborate with a review of literature which also reports a reduction in blood pressure, mean systolic blood pressure ( $-5 \%$ change) and diastolic blood pressure (-2\% change) were lower 5 minutes into hippotherapy if compared with resting conditions before the session in 22 children with spastic cerebral palsy ${ }^{15}$. A study reported all five children with spastic cerebral palsy showed a significantly decreased mean heart rate (mean, 39\%) after an 8-week hippotherapy intervention $^{28}$. Several studies with Animal-Assisted Therapy show a reduction in blood pressure ${ }^{29-31}$.

The increase or decrease in blood pressure and heart rate during some sessions were reported in other studies. A study of veterans with post-traumatic stress disorders showed a decrease in heart rate in only one session, but this was not seen in the other four days of EAT intervention ${ }^{32}$. Another study showed that hippotherapy can benefit children with disabilities attributable to 
Table 6: Heart rateof Normotensive, Hypertensive and Total Group Pre-, during [first ( $\left.1^{\text {st }}\right)$, fifteenth $\left(15^{\text {th }}\right)$, thirtieth $\left(30^{\text {th }}\right)$ minute] and PostEAT (Mean \pm SEM and same letter is statistical significance ${ }^{*} p<0.05,{ }^{* *} p<0.01$ ).

\begin{tabular}{|c|c|c|c|c|c|}
\hline \multicolumn{6}{|c|}{ Normotensive } \\
\hline Day & Pre-EAT & $1^{\text {st }}$ minute & $15^{\text {th }}$ minute & $30^{\text {th }}$ minute & Post-EAT \\
\hline 1 & $78.57 \pm 2.64$ & $79.57 \pm 2.13$ & $79.21 \pm 2.23$ & $79.21 \pm 2.53$ & $77.07 \pm 2.43$ \\
\hline 2 & $79.43 \pm 3.14^{\mathrm{a} \star}$ & $78.21 \pm 2.57$ & $78.86 \pm 2.60$ & $78.43 \pm 2.84$ & $74.86 \pm 3.04^{\mathrm{a} *}$ \\
\hline 3 & $80.00 \pm 2.95$ & $82.57 \pm 2.59$ & $79.79 \pm 2.33$ & $81.57 \pm 4.96$ & $77.36 \pm 1.99$ \\
\hline 4 & $82.79 \pm 2.45$ & $81.36 \pm 2.41$ & $81.50 \pm 2.49$ & $83.43 \pm 2.22$ & $78.64 \pm 2.43$ \\
\hline 5 & $77.71 \pm 2.43$ & $80.43 \pm 3.02$ & $78.93 \pm 2.65$ & $81.57 \pm 3.28$ & $77.64 \pm 2.80$ \\
\hline 6 & $78.71 \pm 3.55$ & $79.57 \pm 2.46^{\mathrm{a} \star}$ & $78.14 \pm 3.00$ & $79.43 \pm 2.72^{b \star}$ & $74.50 \pm 2.91^{a * b *}$ \\
\hline 7 & $79.21 \pm 3.24$ & $80.79 \pm 3.05^{\mathrm{a} *}$ & $78.64 \pm 2.30$ & $79.00 \pm 2.27$ & $75.50 \pm 2.41^{\underline{a} *}$ \\
\hline 8 & $81.79 \pm 2.92$ & $83.43 \pm 3.18$ & $82.43 \pm 3.07$ & $81.93 \pm 2.81$ & $80.50 \pm 2.95$ \\
\hline 9 & $82.71 \pm 3.75^{\mathrm{a} \star}$ & $83.14 \pm 3.66^{b \star *}$ & $81.57 \pm 3.26$ & $81.21 \pm 3.46$ & $77.86 \pm 3.21^{\mathrm{a} * \mathrm{~b} * *}$ \\
\hline 10 & $77.64 \pm 2.97$ & $81.21 \pm 2.80$ & $76.71 \pm 2.99$ & $79.93 \pm 2.75$ & $77.57 \pm 2.99$ \\
\hline \multicolumn{6}{|c|}{ Hypertensive } \\
\hline Day & Pre-EAT & $1^{\text {st }}$ minute & $15^{\text {th }}$ minute & $30^{\text {th }}$ minute & Post-EAT \\
\hline 1 & $78.17 \pm 4.55$ & $69.83 \pm 6.58$ & $77.50 \pm 5.38$ & $71.33 \pm 3.27$ & $73.33 \pm 6.84$ \\
\hline 2 & $74.50 \pm 5.06$ & $71.00 \pm 3.98$ & $74.00 \pm 4.01$ & $72.50 \pm 4.41$ & $70.33 \pm 4.00$ \\
\hline 3 & $68.33 \pm 3.66$ & $68.67 \pm 4.29$ & $72.00 \pm 5.04^{\mathrm{a} *}$ & $69.50 \pm 4.69$ & $65.50 \pm 4.13^{\mathrm{a} *}$ \\
\hline 4 & $69.17 \pm 4.82$ & $71.33 \pm 5.00^{\mathrm{a} *}$ & $68.50 \pm 5.15$ & $69.33 \pm 4.57$ & $65.83 \pm 3.35^{\mathrm{a} *}$ \\
\hline 5 & $68.67 \pm 4.95$ & $70.67 \pm 4.72$ & $72.00 \pm 5.05$ & $71.33 \pm 5.06$ & $66.00 \pm 4.63$ \\
\hline 6 & $66.00 \pm 6.02$ & $70.83 \pm 5.07$ & $67.67 \pm 4.33$ & $70.67 \pm 5.51$ & $65.50 \pm 4.99$ \\
\hline 7 & $73.67 \pm 7.31$ & $77.67 \pm 7.36$ & $70.67 \pm 6.10$ & $73.83 \pm 6.04$ & $74.83 \pm 4.93$ \\
\hline 8 & $78.50 \pm 5.73$ & $72.67 \pm 4.65$ & $71.33 \pm 3.07$ & $70.17 \pm 3.28$ & $68.50 \pm 3.86$ \\
\hline 9 & $82.50 \pm 6.08$ & $72.17 \pm 4.76$ & $75.50 \pm 5.51$ & $73.00 \pm 6.25$ & $73.17 \pm 6.19$ \\
\hline 10 & $77.67 \pm 6.34$ & $73.67 \pm 6.04$ & $70.17 \pm 5.95$ & $74.83 \pm 5.88$ & $72.83 \pm 5.93$ \\
\hline \multicolumn{6}{|c|}{ Total } \\
\hline Day & Pre-EAT & 1st minute & 15th minute & 30th minute & Post-EAT \\
\hline 1 & $78.35 \pm 2.22$ & $76.55 \pm 2.57$ & $78.70 \pm 2.17$ & $76.85 \pm 2.14$ & $75.60 \pm 2.58$ \\
\hline 2 & $77.70 \pm 2.66^{\mathrm{a} \star}$ & $75.75 \pm 2.25$ & $77.10 \pm 2.20$ & $76.55 \pm 2.41$ & $73.45 \pm 2.43^{a *}$ \\
\hline 3 & $77.10 \pm 2.50$ & $79.15 \pm 2.46$ & $78.20 \pm 2.17$ & $78.60 \pm 3.80$ & $74.45 \pm 2.11$ \\
\hline 4 & $79.70 \pm 2.619^{a \star}$ & $78.85 \pm 2.38$ & $78.00 \pm 2.61$ & $79.65 \pm 2.44^{b *}$ & $75.65 \pm 2.30^{\mathrm{a} * \mathrm{~b} *}$ \\
\hline 5 & $74.95 \pm 2.38$ & $77.55 \pm 2.67$ & $77.15 \pm 2.40$ & $78.60 \pm 2.87$ & $74.35 \pm 2.60$ \\
\hline 6 & $75.95 \pm 3.08$ & $77.40 \pm 2.33^{\mathrm{a} \star \star}$ & $75.15 \pm 2.62$ & $77.05 \pm 2.59^{b * *}$ & $72.45 \pm 2.60^{\mathrm{a} * * \mathrm{~b} * *}$ \\
\hline 7 & $78.40 \pm 3.01$ & $80.50 \pm 2.91$ & $76.95 \pm 2.46$ & $77.55 \pm 2.38$ & $75.95 \pm 2.15$ \\
\hline 8 & $81.55 \pm 2.47$ & $80.90 \pm 2.71$ & $79.35 \pm 2.53$ & $79.00 \pm 2.38$ & $77.50 \pm 2.56$ \\
\hline 9 & $83.35 \pm 2.92$ & $80.55 \pm 2.94$ & $80.25 \pm 2.67$ & $79.35 \pm 2.98$ & $78.30 \pm 2.97$ \\
\hline 10 & $78.50 \pm 2.57$ & $79.55 \pm 2.59$ & $75.15 \pm 2.71$ & $78.80 \pm 2.51$ & $77.95 \pm 2.80$ \\
\hline
\end{tabular}

neurological disorders by eliciting an acute autonomic response during the intervention and the recovery period ${ }^{14}$. Limited information exists on the cardiorespiratory responses to EAT in part because of a host of factors that confound measurement precision. Movements of the rider in response to the horse augment the variability. Environmental conditions, such as temperature and humidity, and the logistics of collecting data as the horse and rider move throughout a large space add to the difficulties in characterizing the physical responses to horseback riding ${ }^{15}$.

The low heart rate values maintained during all sessions imply that EAT is a safe physical activity for older individuals. The intensity of the exercise without risk can be calculated from the equation (220 minus the age of the individual). This predictive maximum heart rate (MHR) equation is recognized and applied worldwide ${ }^{33}$. Exercises that fluctuate between $50-60 \%$ of the MHR are considered light, between $61-80 \%$, moderate and between $81-90 \%$, intense $\mathrm{e}^{34}$. Thus, the heart rate oscillation remained on average between $65-83 \mathrm{bpm}(<60 \% \mathrm{MHR})$, deducing that EAT is a light activity. These findings corroborate with previous studies that evaluated the effect of hippotherapy in participants with Down Syndrome $e^{35,36}$. The lowest heart rate averages were for HG. One explanation for this is that antihypertensive drugs, such as beta-adrenergic blockers, may alter baseline heart rate as well as during exercise $\mathrm{e}^{37-39}$. 
Oxygen saturation values remained within normal limits during all sessions, indicating that EAT is safe for normotensive and hypertensive individuals. These findings are consistent with a study about neurological disorders and hippotherapy ${ }^{40}$.

The cardiovascular responses to EAT remain poorly defined. Therefore, this study helps to elucidate its effects in elderly population. Our findings suggest that it is a safe and efficient therapy for this growing population. In addition, EAT is a differentiated intervention in relation to the outpatient and hospital environment. The limitations of this study include few studies on this topic and having a more comprehensive cardiovascular profile. Future research could include cardiorespiratory data integrated with functional tests in order to improve the understanding of physical changes with EAT from a dynamic standpoint.

\section{Conclusions}

Our results suggest that Equine Assisted Therapy can reduce blood pressure in older adults, especially hypertensive individuals.

\section{ACKNOWLEDGEMENTS}

We are grateful for the work of the guides who led the horses during the visits, the elderly people who somehow contributed indirectly to the study, and our coworkers, the horses.

\section{REFERENCES}

1. Miranda GMD, Mendes ACG, Silva ALA. Population aging in Brazil: current and future social challenges and consequences. Rev Bras Geriatr Gerontol. 2016;19(3):507-19. http://dx.doi.org/10.1590/1809-98232016019.150140

2. Beard JR, Officer A, Carvalho IA, Sadana R, Pot AM, Michel JP, et al. The World report on ageing and health: a policy framework for healthy ageing. Lancet. 2016;387(10033):2145-54. http://doi.org/10.1016/S0140-6736(15)00516-4

3. Coutaz M. Hypertension, diabetes, hyperlipidemia: what are the treatment goals for the elderly? Rev Med Suisse. 2017;13(582):1924-30

4. Tan J, Zhang X, Wang W, Yin P, Guo X, Zhou M. Smoking, Blood Pressure, and Cardiovascular Disease Mortality in a Large Cohort of Chinese Men with 15 Years Follow-up. Int J Environ Res Public Health. 2018;15(5):1026. https://doi.org/10.3390/ijerph15051026

5. Bertoluci MC, Moreira RO, Faludi A, Izar MC, Schaan BD, Valerio $\mathrm{CM}$, et al. Brazilian guidelines on prevention of cardiovascular disease in patients with diabetes: a position statement from the Brazilian Diabetes Society (SBD), the Brazilian Cardiology Society (SBC) and the Brazilian Endocrinology and Metabolism Society (SBEM). Diabetol Metab Syndr. 2017;9:53 http://dx.doi.org/10.1186/s13098-017-0251-z

6. Robles NR, Macias JF. Hypertension in the elderly. Cardiovasc Hematol Agents Med Chem. 2015;12(3):136-45. https://doi.org/10.2174/1871525713666150310112350

7. Stafford RS. New High Blood Pressure Guidelines: Back on Track With Lower Treatment Goals, but Implementation Challenges Abound. Am J Prev Med. 2018;55(4):575-8. https://doi.org/10.1016/j.amepre.2018.04.023

8. Levine GN, Allen K, Braun LT, Christian HE, Friedmann E, Taubert $\mathrm{KA}$, et al. Pet ownership and cardiovascular risk: a scientific statement from the American Heart Association. Circulation. 2013;127(23):2353-63. https://doi.org/10.1161/CIR.0b013e31829201e1

9. Silva NB, Osório FL. Impact of an animal-assisted therapy programme on physiological and psychosocial variables of paediatric oncology patients. PLoS One. 2018;13(4):e0194731. https://doi.org/10.1371/journal.pone.0194731
10. Ribeiro MF, Espindula AP, Lage JB, Bevilacqua Júnior DE, Diniz $\mathrm{LH}$, Mello EC, et al. Analysis of the electromiographic activity of lower limb and motor function in hippotherapy practitioners with cerebral palsy. J Bodyw Mov Ther. 2019;23(1):39-47. https://doi.org/10.1016/j.jbmt.2017.12.007

11. Cruz-Fierro N, Vanegas-Farfano M, González-Ramírez MT. DogAssisted Therapy and dental anxiety: a pilot study. Animals (Basel). 2019;9(8):512. https://doi.org/10.3390/ani9080512

12. Araujo TB, Martins WR, Freitas MP, Camargos E, Mota J, Safons MP. An exploration of equine-assisted therapy to improve balance, functional capacity, and cognition in older adults with Alzheimer disease. J Geriatr Phys Ther. 2019;42(3):E155-60. https://doi.org/10.1519/JPT.0000000000000167

13. McDaniel Peters BC, Wood W. Autism and equine-assisted interventions: a systematic mapping review. J Autism Dev Disord. 2017;47(10):3220-42.

https://doi.org/10.1007/s10803-017-3219-9

14. Rigby BR, Gloeckner AR, Sessums S, Lanning BA, Grandjean PW. Changes in cardiorespiratory responses and kinematics with hippotherapy in youth with and without cerebral palsy. Res $Q$ Exerc Sport. 2017;88(1):26-35. https://doi.org/10.1080/02701367.2016.1266458

15. Rigby BR, Grandjean PW. The efficacy of equine-assisted activities and therapies on improving physical function. J Altern Complement Med. 2016;22(1):9-24.

https://doi.org/10.1089/acm.2015.0171

16. Amalakanti S, Pentakota MR. Pulse oximetry overestimates oxygen saturation in COPD. Respir Care. 2016;61(4):423-7. https://doi.org/10.4187/respcare.04435

17. Smith RN, Hofmeyr R. Perioperative comparison of the agreement between a portable fingertip pulse oximeter $\mathrm{v}$. a conventional bedside pulse oximeter in adult patients (COMFORT trial). S Afr Med J. 2019;109(3):154-8. https://doi.org/10.7196/SAMJ.2019.v109i3.13633

18. Nuttall FQ. Body mass index: obesity, bmi, and health: a critical review. Nutr Today. 2015;50(3):117-28. https://doi.org/10.1097/NT.0000000000000092 
19. Diniz LH, Mello EC, Ribeiro MF, Lage JB, Bevilacqua Júnior $D E$, Ferreira $A A$, et al. Impact of hippotherapy for balance improvement and flexibility in elderly people. J Bodywork Mov Therap. 2020;24(2):92-7.

https://doi.org/10.1016/j.jbmt.2019.10.002

20. Mello EC, Regalo SCH, Diniz LH, Lage JB, Ribeiro MF, Bevilacqua Junior DE, et al. Electromyographic analysis of stomatognathic muscles in elderly after hippotherapy. PLoS One. 2020;15(8):e0238036. https://doi.org/10.1371/journal.pone.0238036

21. Associação Nacional de Equoterapia (ANDE-BRASIL). Equoterapia e ciência salvando vidas. Available from: http:// equoterapia.org.br.

22. Merz AA, Cheng S. Sex differences in cardiovascular ageing. Heart. 2016;102(11):825-31 https://doi.org/10.1136/heartjnl-2015-308769

23. Stokar J, Leibowitz D, Durst R, Shaham D, Zwas DR. Echocardiography overestimates LV mass in the elderly as compared to cardiac CT. PLoS One. 2019;14(10):e0224104. https://doi.org/10.1371/journal.pone.0224104

24. Filipovský J. Arterial hypertension in the elderly. Vnitr Lek. 2018;64(11):987-92.

25. Handschin A, Henny-Fullin K, Buess D, Leuppi J, Dieterle T. Hypertension in the elderly. Ther Umsch. 2015;72(6):397-403. https://doi.org/10.1024/0040-5930/a000692

26. Mendonca GV, Pezarat-Correia P, Vaz JR, Silva L, Almeida ID, Heffernan KS. Impact of exercise training on physiological measures of physical fitness in the elderly. Curr Aging Sci. 2016;9(4):240-59. https://doi.org/10.2174/1874609809666160426120600

27. Son WM, Sung KD, Cho JM, Park SY. Combined exercise reduces arterial stiffness, blood pressure, and blood markers for cardiovascular risk in postmenopausal women with hypertension. Menopause. 2017;24(3):262-8. https://doi.org/10.1097/GME.0000000000000765

28. McGibbon NH, Andrade CK, Widener G, Cintas HL. Effect of an equine-movement therapy program on gait, energy expenditure, and motor function in children with spastic cerebral palsy: a pilot study. Dev Med Child Neurol. 1998;40(11):754-62. https://doi.org/10.1111/j.1469-8749.1998.tb12344.x

29. Filan SL, Llewellyn-Jones $\mathrm{RH}$. Animal-assisted therapy for dementia: a review of the literature. Int Psychogeriatr. 2006;18(4):597-611. https://doi.org/10.1017/S1041610206003322

30. McCullough A, Ruehrdanz A, Jenkins MA, Gilmer MJ,Oslon J, Pawar A, et al. Measuring the Effects of an Animal-Assisted Intervention for Pediatric Oncology Patients and Their Parents: A Multisite Randomized Controlled Trial [Formula: see text]. J Pediatr Oncol Nurs. 2018;35(3):159-77.

https://doi.org/10.1177/1043454217748586
31. Krause-Parello CA, Kolassa J. Pet Therapy: Enhancing Social and Cardiovascular Wellness in Community Dwelling Older Adults. J Community Health Nurs. 2016;33(1):1-10.

https://doi.org/10.1080/07370016.2016.1120587

32. Malinowski K, Yee C, Tevlin JM, Birks EK, Dourando MM, PournajafiNazarloo $\mathrm{H}$, et al. The Effects of Equine Assisted Therapy on Plasma Cortisol and Oxytocin Concentrations and Heart Rate Variability in Horses and Measures of Symptoms of Post-Traumatic Stress Disorder in Veterans. J Equine Vet Sci. 2018;64:17-26. https://doi.org/10.1016/j.jevs.2018.01.011

33. Sarzynski MA, Rankinen T, Earnest CP, Leon AS, Rao DC, Skinner $\mathrm{JS}$, et al. Measured maximal heart rates compared to commonly used age-based prediction equations in the Heritage Family Study. Am J Hum Biol. 2013;25(5):695-701. https://doi.org/10.1002/ajhb.22431

34. Arêas GPT, Caruso FCR, Simões RP, Castello-Simões V, Jaenisch RB, Sato TO, et al. Ultra-short-term heart rate variability during resistance exercise in the elderly. Braz $\mathrm{J}$ Med Biol Res. 2018;51(6):e6962.

http://dx.doi.org/10.1590/1414-431×20186962

35. Bevilacqua Junior DE. Avaliação cardiovascular, muscular e da mobilidade funcional de adolescentes com síndrome de Down atendidos na Equoterapia e Fisioterapia [dissertation]. [Uberaba]: Universidade Federal do Triângulo Mineiro; 2018.

36. Bevilacqua Junior DE, Lopes LLM, Accioly MF, Ribeiro MF, Ferreira AA, Teixeira VPA, et al. Avaliação da modulação autonômica em indivíduos com síndrome de Down na equoterapia.ConScientiae Saude. 2016;15(3):433-9. https://doi.org/10.5585/conssaude.v15n3.6360

37. Naci H, Salcher-Konrad M, Dias S, Blum MR, Sahoo SA, Nunan D, et al. How does exercise treatment compare with antihypertensive medications? A network meta-analysis of 391 randomized controlled trials assessing exercise and medication effects on systolic blood pressure. Br J Sports Med. 2019;53(14):859-69. https://doi.org/10.1136/bjsports-2018-099921

38. Godlasky E, Hoffman T, Weber-Peters S, Bradford R, Miller N, Kunselman AR, et al. Effects of $\beta$-Blockers on Maximal Heart Rate Prediction Equations in a Cardiac Population. J Cardiopulm Rehabil Prev. 2018;38(2):111-7. http://dx.doi.org/10.1097/HCR.0000000000000328

39. Roberts PA, Lin ACW, Cowan BR, Young AA, Stewart R Comparison of effects of losartan and metoprolol on left ventricular and aortic function at rest and during exercise in chronic aortic regurgitation. Int J Cardiovasc Imaging. 2018;34(4):615-24. http://dx.doi.org/10.1007/s10554-017-1268-y

40. Cabiddu R, Borghi-Silva A, Trimer R, Trimer V, Ricci PA, Monteiro $\mathrm{Cl}$, et al. Hippotherapy acute impact on heart rate variability non-linear dynamics in neurological disorders. Physiol Behav. 2016;159:88-94.

https://doi.org/10.1016/j.physbeh.2016.03.012 